\title{
Erratum to: Knowability as potential knowledge
}

\section{André Fuhrmann}

\section{Erratum to: Synthese DOI 10.1007/s11229-013-0340-y}

Page 7 [online version], lines 10-16 ("Clearly, [...] Thus we have") should be replaced by:

If $B$ is true under the specification [A], then whatever is true under the conjunctive specification $[A \wedge B]$ must already be true under $[A]$ alone:

$$
[A] B \rightarrow([A \wedge B] C \rightarrow[A] C) .
$$

The converse of the consequent holds under the same antecedent-condition: Suppose that all worlds specified in a given world by the description $A$ satisfy the condition $B$. Then the conjunctive specification $[A \wedge B]$ cannot be more restrictive than $[A]$, for $[A]$ alone already restricts the selected worlds to $B$-worlds. Thus we have $[\ldots]$

The online version of the original article can be found under doi:10.1007/s11229-013-0340-y.

\footnotetext{
A. Fuhrmann $(\varangle)$

Institut für Philosophie, Goethe-Universität Frankfurt am Main, 60629 Frankfurt, Germany

e-mail: fuhrmann@em.uni-frankfurt.de
} 